\title{
Future inhaled drugs by virtual innovation: allergen delivery inhibitors
}

\author{
“...inhaled therapy has obvious attractions in neutralizing 'foreign' threats and offers an \\ interesting creative freedom for chemical design."
}

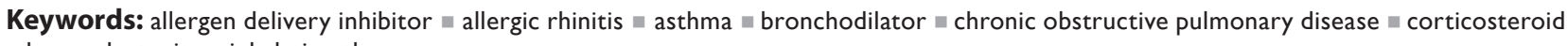
w house dust mite $=$ inhalation therapy

It is a common misconception that drug delivery to the airways provides an easy development option for agents where a legitimate choice between topical or systemic administration exists. In an inhaled drug-discovery project the attraction of a chemistry strategy, which can be antithetical to Lipinski principles is obvious, but the scientific hurdles and costs are typically relocated rather than retired. For respiratory franchise holders in big pharmaceutical companies some of the unique challenges posed by inhalation may now be seen as routine. However, the expertise underpinning them is specialized, cultivated over years of investment, concentrated in a few locations, largely invisible to the public domain and poorly supported by specific academic training. This makes an inhaled R\&D capability an asset, which is difficult to replicate.

Existing inhaled medicines treat, but do not cure, diseases such as asthma, rhinitis and chronic obstructive pulmonary disease (COPD), the prevalence of which continues to increase with profound socioeconomic impacts. These medicines are commercially successful. The global respiratory market is worth approximately US $\$ 60$ billion and has been interestingly resistant to massive erosion by generics, despite significant patent expiries [1]. In the golden circle of big pharmaceutical companies, established respiratory franchises form solid foundations and have provided engines of growth for smaller companies that have exploited opportunities to develop blockbuster presence in areas of unmet need. These considerations notwithstanding, there is a danger that the capability to fully exploit inhaled drug delivery in the future will be undermined by the unprecedented turmoil as pharmaceutical companies struggle to redescribe its identity and function, especially if the true scientific and commercial asset values of the inhalation niche are not recognized.

\section{Why inhalation?}

Intuitively, respiratory medicines should be delivered directly to their sites of action in the airways, thereby minimizing systemic off-target actions, yet not all are. Front-line therapies act at receptors expressed throughout the body so an important reason justifying their inhaled delivery is actually the need to minimize systemic on-target actions rather than to maximize efficacy.

Inhalation is a challenging delivery option for small biotechnology companies or virtual teams whose drug-discovery models are 'lean' approaches to an eventual out-licensing exit. Usually, without a predefined delivery device or development partner, the candidate drug strategy must accommodate the widest number of options and provide reassurance regarding developability for inhalation, probably in combination with other products. The program must be achievable with in-house resource or from a contract sector where the relevant expertise is, at best, highly fragmented. Does this mean that the inhaled route is in reality a 'no-go' area for innovative entrants? If that were to be the case, would it matter? To answer both questions requires an examination of why medicines are given by inhalation and some speculation about future needs and opportunities.

The obvious unmet need in existing respiratory therapy is the failure of existing approaches to stem the rising tide of disease. By 2015, in the seven major markets the prevalence of asthma is projected to be $7.2 \%$, COPD $8.9 \%$ and allergic rhinitis $22.8 \%$ [1]. The high prevalence of allergic rhinitis is a significant figure in this profile. Although frequently discounted as a trivial condition, and, hence, a low priority for pharmaceutical innovation, there is now comprehensive evidence that it is a major risk identifier for the subsequent development of asthma, the so-called 'allergic march' [2-4]. A major therapeutic opportunity,

\section{Clive Robinson ${ }^{\dagger 1}$, Jihui Zhang', David R Garrod $^{2}$, Gary K Newton ${ }^{3}$, Kerry Jenkins ${ }^{3}$ \& Trevor R Perrior ${ }^{3}$}

'Division of Biomedical Sciences, St George's, University of London, London, SWI7 ORE, UK

${ }^{2}$ Faculty of Life Sciences, University of Manchester, Manchester, MI3 9PT, UK

${ }^{3}$ Domainex Ltd, 162 Cambridge Science Park, Cambridge, CB4 0GH, UK

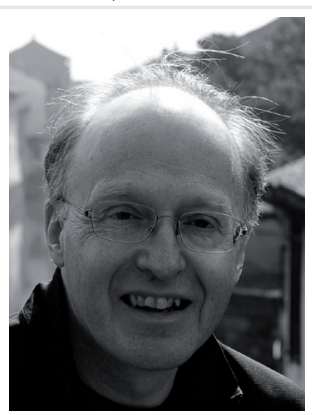

${ }^{\dagger}$ Author for correspondence: Tel.: +44 2087255620 Fax: +44 2087250685 E-mail: c.robinson@sgul.ac.uk 
therefore, exists, but its exploitation for patient benefit will require innovative approaches to target selection, an inhaled drug capability and a revision to business thinking.

Historically, most effort in respiratory therapeutics has been directed towards asthma, but recently there has been a concentration on COPD, initially exploiting repurposing opportunities with asthma products, but now with a diversity of approaches under trial. The drug treatment of both conditions is circumscribed by treatment guidelines (step therapy) in which 'reliever' medications (typically $\beta_{2}$ agonists in asthma and antimuscarinics in COPD), and anti-inflammatory corticosteroids occupy center stage. Since the launch in 1999 of Seretide ${ }^{\mathrm{TM}} /$ Advair $^{\circledR}$, the first combined bronchodilator and inhaled corticosteroid (ICS), there has been a steady emphasis on the development and clinical use of combination products rather than monotherapy. In the case of Seretide/Advair, the active ingredients are fluticasone propionate and the long-acting $\beta_{2}$ agonist (LABA) salmeterol. This combination has proved to be clinically useful, financially successful and has led to emulation, for example, budesonide/formoterol and beclomethasone/ formoterol, with several others currently in development to offer potential additional benefits and to cover patent expiries. In 2009, the global sales of combination ICS/LABAs were greater than the aggregate for ICS, LABA or short-acting $\beta_{2}$ agonist monotherapies [1]. This move to combinations has been driven by clinical efficacy and the corresponding benefit of potentially using only a single inhaler, which is liked by patients, and more definitively by regulatory actions that effectively signal the end for LABA monotherapy because of safety concerns. The inhaled combination therapy approach is equally valid in COPD. Existing ICS/LABA combinations developed for asthma therapy are increasingly used in COPD and are expected to be joined by combinations of LABAs with longacting muscarinic antagonists. A further evolution in combination therapy is embodied in muscarinic antagonist $-\beta_{2}$ agonists. Optimizing actions at multiple targets within a single molecule is a challenging medicinal chemistry assignment, but one that has attractions for simplified development. Multifunctional drugs are more likely to be anti-Lipinski in nature because achieving potency and selectivity at multiple targets will influence the size of molecules that can be designed. Inhalation is, therefore, an ideal method of delivery for such agents, provided that the physical properties for inhalation can be assured.

Despite not stemming the rising tide of respiratory disease, the core therapy in asthma and COPD of bronchodilators and ICS is life-saving in emergency and has the potential to improve quality of life through symptom relief. But surely more is possible? However, more than 30 years' endeavor by pharmaceutical companies, largely directed at downstream effectors, has failed to produce interventions that supplant the dominance of $\beta_{2}$-agonists and ICS, either because they lack efficacy (act too far downstream in a high-redundancy process) or, as is the case for biologicals, are beset by other issues. Does this mean that the treatment of asthma is consigned to be of slow evolution rather than revolution, a reason for pessimism among asthma sufferers? Such a downbeat analysis about future asthma therapy might be legitimate given the early-stage focus on COPD where arguably a greater market potential exists from only modest innovation, a safe harbor for a storm-tossed industry. This need not be the case.

The most effective treatment for these diseases is to identify their triggers and eliminate or limit exposure to them. For COPD, cessation of tobacco smoking has the greatest impact on the decline in respiratory function, so the decisive healthcare intervention is nonpharmacological. For conditions such as allergic asthma and rhinitis the situation is interestingly different and amenable to radically new pharmacological strategies exploiting a real advantage of inhalation. One such example comes from the Wellcome Trust's Seeding Drug Discovery Initiative, which has pursued the blockade of a major disease trigger. This approach originates from research into the molecular basis of allergenicity and, if successful, creates the possibility for disease modification in ways that cannot be achieved by existing medicines.

\section{- Allergen delivery inhibitors: a new approach to asthma and rhinitis}

House dust mites (HDM) are globally prevalent, potently allergenic and are persistent triggers of rhinitis and asthma. A family of cysteine peptidases forming the Group I HDM allergens is clinically the most significant initiator of such attacks. These peptidases (e.g., Der p 1, Der f 1 and Eur $\mathrm{m} \mathrm{I}$ ) are digestive enzymes in HDM and are excreted in faecal pellets, which are of a respirable size for humans. When the pellets impact 
on the airway lining the peptidase allergens are liberated alongside nonpeptidase bystander allergens. The cysteine peptidases cause the development, maintenance and escalation of allergic disease because they have actions that facilitate the delivery of allergens to antigen-presenting cells and that trigger signaling pathways, which promotes allergy-biased immune responses (for review see [5]). These peptidase-mediated events form part of the innate (IgE-independent) response to allergens, an aspect of immunity now recognized for its importance in the development of allergy. Peptidase allergens contribute to innate immunity by various mechanisms. These include the cleavage of epithelial tight junctions to facilitate allergen delivery and cell migration [5], the cleavage of receptors on airway cells to elicit the release of cytokines (e.g., IL-6, GM-CSF) that promote allergic bias [6], and of chemokines (e.g., CCL2, CCL5, CCL20 and IP-10) that cause recruitment of dendritic cells to the airways for antigen capture and presentation to T-lymphocytes [7]. Peptidase allergens activate mast cells by IgE-independent mechanisms, they degrade antipeptidase defenses (which may already be defective in allergy) [8] and they degrade other potential protective factors such as surfactant proteins [9].

Conveniently forming a single therapeutic target because of their highly conserved common molecular structure, they constitute a novel sub-family of cysteine peptidases, which can be structurally distinguished from potential mammalian off-targets. Detailed knowledge of the peptidase allergen target facilitated a structurebased drug-design program leading to successful candidate nomination. Reflecting a key action of peptidase allergens, these new drugs are known as allergen delivery inhibitors (ADIs). The fact that the nonhuman target is itself inhaled creates a justification for topical delivery, which is notably different from that of existing asthma medicines. The desire to maximize clinical benefit and simplify treatment regimens provides a further argument that an inhaled ADI combination therapy would be highly desirable.

The profile of an inhaled ADI is an agent that combines target potency and selectivity with a maximization of lung residence time. To provide protection against the inhaled target, but without the need to exert systemic action, ADI design, therefore, explored anti-Lipinksi attributes such as low permeability, low bioavailability, high clearance and high plasma protein binding, to minimize systemic exposure and enhance lung retention. For inhaled drugs, controlling retention (e.g., by slow off-rate, low permeability, low solubility, tissue binding) is important in achieving dosing regimens, which will be convenient for patients and in providing compatibility with the other component of a binary combination. Expediently, the required kinetic properties are achievable from chemical scaffolds, such as peptides, often disfavored in oral programs. Peptides are likely entry points in any peptidase inhibitor campaign such as the ADI program, but these features need to be combined with physical properties (crystallinity, low hygroscopicity, thermal stability and micronisability) that suit the intended delivery device(s) and allow the production of reproducible particles for delivery to the lungs. Due regard must be given to achieving the best balance of these features to ensure developability. For example, experience teaches that achieving an enduring action by only engineering compounds to have low solubility is likely to result in an irritancy nuisance.

Compounds arising from the ADI program show excellent efficacy in disease models when delivered to the airways. The availability of the first selective agents aimed at this target family means that this unique approach could be used as either monotherapy or combination therapy in asthma and rhinitis. One possibility for some interesting combination options, especially in persistent rhinitis, is to exploit a broad palette of reliever medications in tandem with an ADI to create a disease-modifying approach that reduces acute symptoms while, simultaneously, halting the allergic march and preventing escalation into asthma.

\section{Future risks, future opportunities}

The future of respiratory therapy is an interesting microcosm of the unplanned impacts arising from the turbulence in global pharmaceutical companies. With a need to treat complex chronic conditions the therapeutic area is undeniably challenging and is not aided by commercial perceptions based on misinterpretations of success rates biased by repetitious use of a flawed strategy to seek new therapies. However, it is reasonable for patients, particularly those with asthma and allied disorders, to have an expectation that better treatments should become available given the research investment in the basic and clinical sciences underlying these diseases. Inhaled therapy has additional potential for significant growth in the treatment of respiratory infections. 
These can be primary causes of disease (e.g., TB) or important exacerbating factors in others (e.g., cystic fibrosis, asthma and COPD), and it is surprising that this has not been pursued with greater imagination and vigor. The therapeutic targets in these examples enter by the respiratory tract and are harbored in the lungs. Therefore, as in the ADI approach, inhaled therapy has obvious attractions in neutralizing 'foreign' threats and offers an interesting creative freedom for chemical design.

Potential obstacles to healthcare progress exist, among them the growing lack of diversity among big pharmaceutical companies and capability gaps arising from 'slash and burn' restructuring and a growing reliance on externally originated candidates. The pipeline of ideas is not the major problem, but strategic gap funding is necessary to bring forward the innovative programs that are required to meet healthcare needs and pharmaceutical company pipelines alike. The Wellcome Trust's Seeding Drug Discovery Initiative program is a bold example of such an intervention that allows virtual teams or small companies to contribute to the innovation pipeline. However, this is insufficient of itself. The fragmentation of early-stage work creates an R\&D landscape in which exciting opportunities exist, but there are emerging threats as specialized capabilities are endangered by restructuring. Therefore, it is imperative for the advancement of drug research that this hardwon expertise is retained, for example in the contract research sector which is increasingly taking on the responsibility for many aspects of drug discovery and development. The niche area of inhaled drugs is one clear example where this is necessary if it is to thrive in a new drugdiscovery environment that is reliant on small or virtual innovators. Fragmentation of expertise as it migrates into the contract sector must be avoided otherwise healthcare opportunities may be lost.

\section{Financial \& competing interests disclosure}

The authors have no relevant affiliations or financial involvement with any organization or entity with a financial interest in or financial conflict with the subject matter or materials discussed in the manuscript. This includes employment, consultancies, honoraria, stock ownership or options, expert testimony, grants or patents received or pending, or royalties.

No writing assistance was utilized in the production of this manuscript.

\section{Bibliography}

1 Business Insights Ltd. The Asthma, COPD and Allergic Rhinitis Market Outlook to 2015. 1-104 (2010).

2 Cruz AA, Popov T, Pawankar R et al. Common characteristics of upper and lower airways in rhinitis and asthma: ARIA update, in collaboration with GA(2)LEN. Allergy 62(Suppl. 84) 1-41 (2007).

3 Danielsson J, Jessen M. The natural course of allergic rhinitis during 12 years of follow-up. Allergy 52, 331-334 (1997).

4 Guerra S, Sherrill DL, Martinez FD, Barbee RA. Rhinitis as an independent risk factor for adult-onset asthma. J. Allergy Clin. Immunol. 109, 419-425 (2002).
5 Stewart GA, Zhang J, Robinson C. The structure and function of allergens. In: Middleton's Allergy. Principles and Practice. Adkinson NF, Bochner BS, Busse WW, Holgate ST, Lemanske RF, Simons FER (Eds). Mosby, PA, USA 569-608 (2009).

6 Asokananthan N, Graham PT, Stewart DJ et al. House dust mite allergens induce proinflammatory cytokines from respiratory epithelial cells: the cysteine protease allergen, Der $\mathrm{p} 1$, activates protease-activated receptor (PAR)-2 and inactivates PAR-1. J. Immunol. 169, 4572-4578 (2002).

7 Pichavant M, Charbonnier AS, Taront $S$ et al. Asthmatic bronchial epithelium activated by the proteolytic allergen Der $\mathrm{p} 1$ increases selective dendritic cell recruitment. J. Allergy Clin. Immunol. 115, 771-778 (2005).
8 Brown A, Farmer K, MacDonald L et al. House dust mite Der p 1 downregulates defenses of the lung by inactivating elastase inhibitors. Am. J. Respir. Cell Mol. Biol. 29, 381-389 (2003).

9 Deb R, Shakib F, Reid K, Clark H. Major house dust mite allergens Dermatophagoides pteronyssinus 1 and Dermatophagoides farinae 1 degrade and inactivate lung surfactant proteins A and D. J. Biol. Chem. 282, 36808-36819 (2007). 